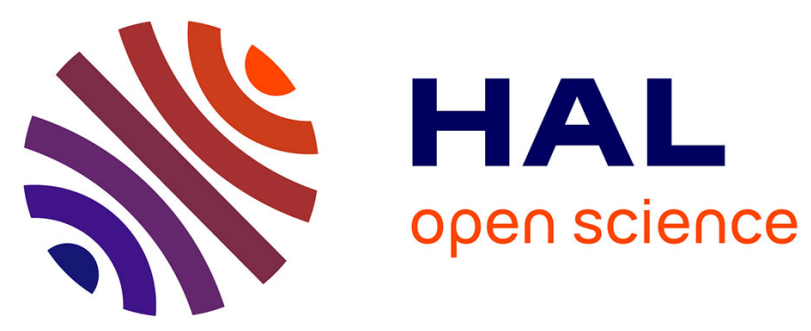

\title{
The effect of water suppression on the hepatic lipid quantification, as assessed by the LCModel, in a preclinical and clinical scenario
}

Amandine Coum, Fanny Noury, Elise Bannier, Karima Begriche, Bernard Fromenty, Yves Gandon, Hervé Saint-Jalmes, Giulio Gambarota

\section{To cite this version:}

Amandine Coum, Fanny Noury, Elise Bannier, Karima Begriche, Bernard Fromenty, et al.. The effect of water suppression on the hepatic lipid quantification, as assessed by the LCModel, in a preclinical and clinical scenario. Magnetic Resonance Materials in Physics, Biology and Medicine, 2016, 29 (1), pp.29-37. 10.1007/s10334-015-0508-1 . hal-01237087

\section{HAL Id: hal-01237087 \\ https://hal-univ-rennes1.archives-ouvertes.fr/hal-01237087}

Submitted on 6 Jul 2016

HAL is a multi-disciplinary open access archive for the deposit and dissemination of scientific research documents, whether they are published or not. The documents may come from teaching and research institutions in France or abroad, or from public or private research centers.
L'archive ouverte pluridisciplinaire HAL, est destinée au dépôt et à la diffusion de documents scientifiques de niveau recherche, publiés ou non, émanant des établissements d'enseignement et de recherche français ou étrangers, des laboratoires publics ou privés. 


\title{
The effect of water suppression on the hepatic lipid quantification, as assessed by the LCModel, in a preclinical and clinical scenario.
}

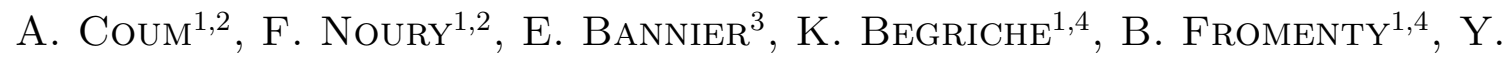 \\ Gandon $^{3}$, H. SAint-Jalmes ${ }^{1,2,5}$ And G. Gambarota ${ }^{1,2}$ \\ ${ }^{1}$ Université de Rennes 1, Rennes F-35000, France \\ ${ }^{2}$ LTSI, INSERM, UMR 1099, Rennes F-35000, France \\ ${ }^{3}$ Département de Radiologie, CHU de Rennes, Rennes F-35000, France \\ ${ }^{4}$ INSERM U991, Rennes F-35000, France \\ ${ }^{5}$ CRLCC, Centre eugène Marquis, Rennes F-35000, France
}

\begin{abstract}
Object To investigate the effect of water suppression on the hepatic lipid quantification, using the LCModel.

Material and Methods MR spectra with and without water suppression were acquired in the liver of mice at $4.7 \mathrm{~T}$ and patients at $3 \mathrm{~T}$, and processed with the LCModel. The Cramér-Rao Lower Bound (CRLB) values of the seven lipid resonances were determined to assess the impact of water suppression on hepatic lipid quantification. A paired t-test was used for comparison between the CRLBs obtained with and without water suppression.

Results For the preclinical data, in the high (low) fat fraction subset an overall impairment in hepatic lipid quantification, i.e. an increase of CRLBs, (no significant change of CRLBs) was observed in spectra acquired with water suppression. For the clinical data, there were no substantial changes in the CRLB with water suppression. Because i) the water suppression does not overall improve the quantification of the lipid resonances and ii) the MR spectrum without water suppression is always acquired for fat fraction calculation, the optimal data-acquisition strategy for liver MRS is to acquire only the MR spectrum without water suppression.

Conclusion For quantification of hepatic lipid resonances, it is advantageous to perform MR spectroscopy without water suppression in a clinical and preclinical scenario (at moderate fields).
\end{abstract}




\section{Introduction}

Obesity is rapidly becoming one of the major health concerns worldwide. An increasing morbidity and mortality connected with many obesity-related diseases, including type-2 diabetes, hypertension and non-alcoholic fatty liver disease (NAFLD) have been observed in recent years [1]. Fuelled by this worldwide health problem, there has been a growing interest in lipid metabolism, both in the clinical and research settings, with particular focus on the quantification of lipids in liver [2-4].

For many years, biochemical and histochemical analyses of biopsies have been the gold standard for the lipid quantification. In the last decade, magnetic resonance imaging (MRI) and spectroscopy (MRS) have emerged as the methods of choice for assessment of lipid content (i.e., fat fraction (FF)) in liver [5-8]. The clear advantage of MR techniques over biopsies is that the MRI and MRS are noninvasive, thus can be performed repeatedly in longitudinal studies without risk of complications [9]. MRI and MRS provide complementary information regarding hepatic lipids. Using MRI, the FF can be determined over the whole liver volume, whereas with MRS the FF as well as lipid composition can be measured [10] - only however over a limited volume with typical dimensions of $2 \times 2 \times 2 \mathrm{~cm}^{3}$ in clinical studies. The assessment of lipid composition is achieved by measuring the resonance peaks of each lipid component, that is the methylene $\left(\left(\mathrm{CH}_{2}\right)_{n}\right)$ protons, the methyl $\left(\mathrm{CH}_{3}\right)$ protons, the olefinic protons, the $\alpha$-methylene to carboxyl protons, the $\beta$-methylene to carboxyl protons, the $\alpha$-olefinic protons and the diacyk protons [6].

The knowledge of lipid composition is essential for investigations of lipid metabolism and, in the last decade, MRS has been used to this purpose in many preclinical and clinical studies. From a dataacquisition point of view, two main approaches can be distinguished in MRS: one where, prior to excitation, water suppression pulses are used and the other where no water suppression is applied. To date, however, no study has systematically and quantitatively investigated the effect of water suppression on hepatic lipid quantification. For MR spectra, a means to determine the accuracy in the quantification of resonance peaks is provided by the Cramér-Rao Lower Bound (CRLB). Thus, the CRLB calculated for each lipid resonance can be used as a metric when comparing the different data-acquisition strategies.

Given the relevance of lipid composition in current research, it is of interest to determine the optimal data-acquisition strategy in order to improve the quantification of hepatic lipids, in both preclinical and clinical studies. The aim of this study was to investigate the effect of water suppression on the quantification of lipid resonances in liver, as assessed by the LCModel [11], in a pre-clinical and a clinical scenario. Data analysis was performed on MR spectra acquired in vivo at $4.7 \mathrm{~T}$ in mice and at $3 \mathrm{~T}$ in patients.

\section{$2 \quad$ Material and methods}

\subsection{Animals and Patients}

Fifteen c57BL/6JRj female mice were used for the current study, including nine obese (ob/ob and $\mathrm{db} / \mathrm{db}$ ) mice (Janvier Labs, Le Genest-Saint-Isle, France). The animals were five weeks old. For MR experiments, animals were anaesthetized by intra-peritoneal injection of ketamine $(55 \mathrm{mg} / \mathrm{kg})$ 
and xylazine $(5.5 \mathrm{mg} / \mathrm{kg})$. MR measurements on each mouse were performed at different time points (day 0,14 and 28) in order to obtain spectra with different levels of hepatic fat overload, from smallmoderate fatty infiltration to severe fatty infiltration.

Eleven patients (age $=49 \pm 12$ years) suspected of presenting hepatic fat overload were included in the clinical study. All preclinical and clinical experiments were approved by the Animal Ethics Committee and Institutional Review Board, respectively.

\subsection{In vivo ${ }^{1} \mathrm{H}$ MRS}

MR experiments on mice were performed using a $4.7 \mathrm{~T}$ MR scanner (47/40 USR Bruker Biospec, Bruker BioSpin GmbH, Ettlingen, Germany) with a BGA12 gradient system (maximum strength of $400 \mathrm{mT} / \mathrm{m}$ ) and a birdcage coil (72 mm inner-diameter). During experiments, the mouse respiration rate was monitored using a fiber-optic pneumatic pillow (SA Instruments, Inc., Stony Brook, NY, USA) to synchronize the acquisition with the respiratory frequency. Multi-slice T1-weighted RARE images (RARE: Rapid Acquisition with Relaxation Enhancement [12]) were acquired for anatomical localization, using the following parameters: $\mathrm{TR}=900 \mathrm{~ms}, \mathrm{TE}=11.7 \mathrm{~ms}, 20$ slices, a slice thickness of $1 \mathrm{~mm}$, a field of view (FOV) of $5 \times 5 \mathrm{~cm}^{2}$, a matrix of $192 \times 192$ and 3 signal averages. MRS was performed using the double spin-echo Point REsolved SpectroScopy (PRESS) sequence [13] in a voxel of interest (VOI) of $3 \times 3 \times 3 \mathrm{~mm}^{3}$ positioned in the right lobe of the mouse liver. For each mouse, two MR spectra were acquired: i) one spectrum without water suppression ( $\mathrm{TR}=4000 \mathrm{~ms}$, $\mathrm{TE}=11 \mathrm{~ms}, 64$ signal averages $)$ and ii) one spectrum with water suppression $(\mathrm{TR}=2500 \mathrm{~ms}, \mathrm{TE}=11 \mathrm{~ms}, 64 / 256$ signal averages (obese/control mice)). The VAPOR water suppression module was used (VAPOR: Variable pulse Power and Optimized Relaxation delays [14]) with a spectral bandwidth of $150 \mathrm{~Hz}$. Manual adjustment of the water suppression pulse gains was performed on each mouse to optimize the suppression of the water resonance.

MR experiments on patients were performed using a 3 T MR scanner (Verio, Siemens Medical Solutions, Erlangen, Germany) with a 32-channel torso coil. Multi-slice T2-weighted HASTE images (HASTE: Half-Fourier Acquisition Single-shot Turbo Spin Echo [15]) were acquired for anatomical localization, using the following parameters: $\mathrm{TR}=1000 \mathrm{~ms}, \mathrm{TE}=96 \mathrm{~ms}, 30$ slices, a slice thickness of $5 \mathrm{~mm}$, FOV of $38 \times 28 \mathrm{~cm}^{2}$ and an in-plane resolution of $1.5 \times 1.2 \mathrm{~mm}^{2}$. MRS was performed using the STimulated Echo Acquisition Mode (STEAM) sequence [16] in a VOI of 30x30x30 $\mathrm{mm}^{3}$ placed in the right lobe of the liver avoiding large vessels. Similarly to the preclinical study, for each patient two MR spectra were acquired: i) one spectrum without water suppression and ii) one spectrum with water suppression. The water suppression module was the WET water suppression (WET: Water suppression Enhanced through T1 effect [17]) and all MRS acquisitions were performed under breath hold, with $\mathrm{TR}=4000 \mathrm{~ms}, \mathrm{TE}=20 \mathrm{~ms}$ and 2 signal averages.

\subsection{Analysis of MRS data and statistical analysis}

All MR spectra were processed with the LCModel (Version 6.3-0L, Stephen Provencher, Oakville, ON, Canada) without introduction of prior knowledge. Nine lipid resonances were identified, seven of those lipid resonances were used in the current study, as illustrated in Figure 1. The Lip13 resonance area 
(originating from the $(\mathrm{CH} 2) n$ protons at $1.3 \mathrm{ppm}$ ) represents the major resonance and accounts for approximately $70 \%$ of the total lipid signal. For this reason, it is often used to quantify the fat content. The other lipid resonances - with the exception of the Lip41 and Lip43 resonances (at 4.1 and 4.3 ppm, respectively) corresponding to the glycerol chain- provide information on the fat composition [18-20]. The LCModel employs a flexible combination of Gaussian and Lorentzian lineshapes to model the lipid resonances. For each resonance, the LCModel provides i) the 'concentration', measured as the resonance area (also commonly referred to as 'peak area' in the case of non-overlapping signals) and ii) the Cramér-Rao Lower Bound (CRLB) [21], which yields an estimate of measurement reliability. It should be noted that, when using the LCModel basis set for fat quantification, the user has no access to the individual curve fitting of each resonance. In all calculations, only the resonances displaying a CRLB $<10 \%$ were considered.

The fat fraction (FF) was calculated from the MR spectra acquired without water suppression as the ratio of the Lip13 resonance area ([Lip13]) relative to the sum of the water and Lip13 resonances areas [4]. Preclinical and clinical spectra were each separated into two subsets considering an FF threshold of $15 \%$ : the preclinical (clinical) subset with a high fat fraction (HFF) and the preclinical (clinical) subset with a low fat fraction (LFF). A new parameter ' $\mathrm{R}$ ' was introduced in order to assess the impact of the water suppression on the Lip53+52 resonance, which is very close to the water resonance. $\mathrm{R}$ was defined as the ratio of the resonance area of the Lip13 over the Lip $53+52$, that is, $R=[\operatorname{Lip} 13] /[\operatorname{Lip} 53+52]$. The parameter $\mathrm{R}$ was evaluated in spectra with and without water suppression. In the ideal case, the value of $R$ would not change with the water suppression. An increase of $R$ would indicate a reduction of the signal of the Lip53+52 due to the RF pulses of water suppression module. This parameter was calculated for preclinical and clinical data, only for spectra presenting a CRLB $¡ 10 \%$ for the Lip $53+52$ resonance.

In order to assess the effect of water suppression on the quantification of lipid resonances, a paired t-test was used for comparison between the CRLBs of lipid resonances obtained with and without water suppression. The level of significance was set at 5

\section{Results}

\subsection{In vivo ${ }^{1} \mathrm{H}$ MRS}

Figure 1 shows an example of a non-water-suppressed (noWS) and a water-suppressed (WS) spectrum acquired on a mouse liver with severe fatty infiltration (Figure 1, A and B, respectively), and on a mouse liver with no fatty infiltration (Figure 1, C and D, respectively). Each lipid resonance is indicated by the arrow on the non-water-suppressed spectrum on the mouse liver with severe fatty infiltration (Figure 1, A). In the two spectra acquired on the mouse liver with severe fatty infiltration, a similar spectral quality of the lipid resonances can be observed (Figure 1, A and B). In the spectra acquired on the mouse liver without fatty infiltration (Figure 1, C and D), the major lipid resonance (Lip13) is the only lipid resonance well above the noise level. An excellent water suppression was obtained with the VAPOR water suppression module in combination with the manual adjustment of 
the water suppression pulse gains (Figure 1, B and D).

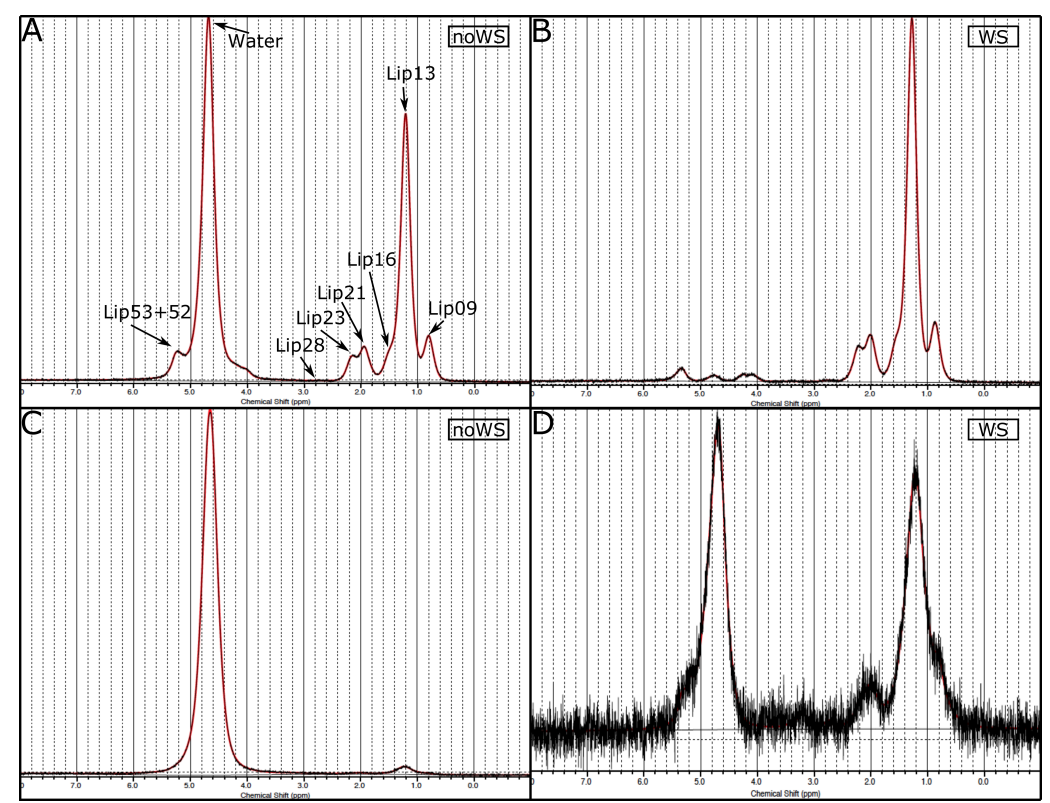

Figure 1: ${ }^{1} H$ MR spectra acquired on a mouse liver with a high fat fraction (FF=36.5\%) without and with water suppression ( $A$ and $B$, respectively), and on a mouse liver with a low fat fraction ( $F F=2.0 \%$ ) without and with water suppression ( $C$ and $D$, respectively). Each lipid resonance is indicated by the arrows on the spectrum without water suppression (noWS) acquired on the mouse with high fat fraction. An efficient suppression of the water resonance (spectra WS) was achieved with the VAPOR method.

Figure 2 shows a comparison of an MR spectrum acquired on a mouse liver at $4.7 \mathrm{~T}$ (Figure 2, A) and on a patient liver at $3 \mathrm{~T}$ (Figure 2, B) with moderate fatty infiltration. In these two spectra, the $\mathrm{FF}$ is comparable and approximately $15 \%$. The preclinical and clinical spectra display similar spectral features, validating the idea that similar acquisition strategies can be used for preclinical and clinical MRS acquisitions on liver at these field strengths.
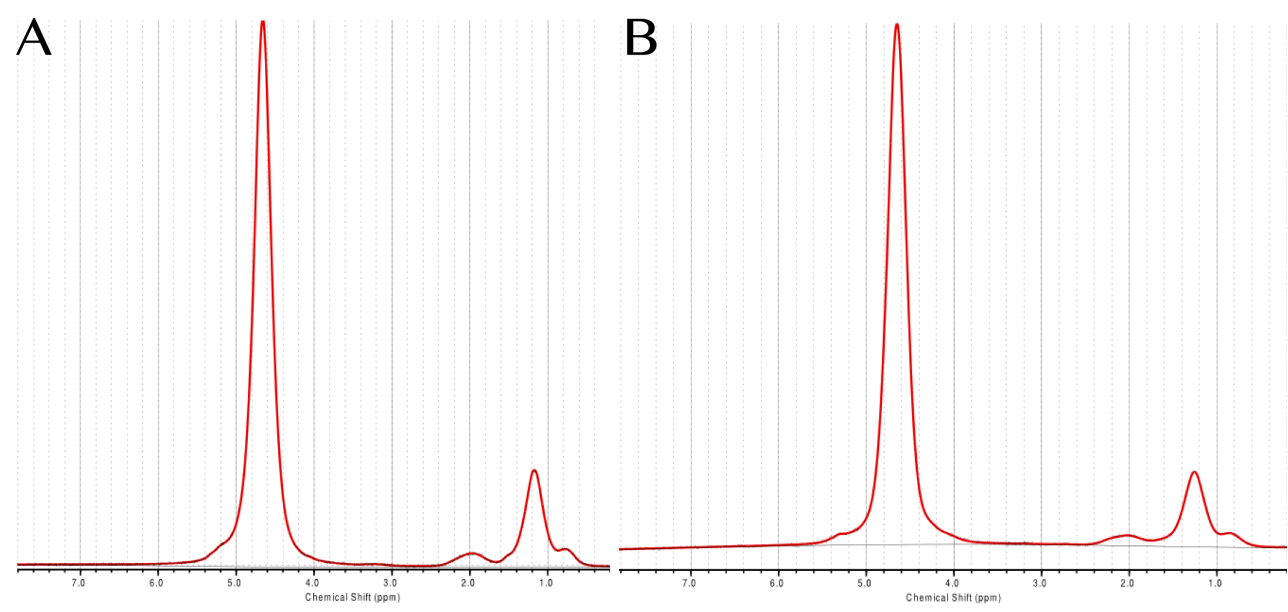

Figure 2: ${ }^{1} \mathrm{H}$ MR spectra processed with the LCModel, acquired (A) at $4.7 \mathrm{~T}$ on a mouse liver with a fat fraction of $15.0 \%$ and $(B)$ at $3 \mathrm{~T}$ on a patient liver with a fat fraction of $13.0 \%$. 


\subsection{Analysis of MRS data and statistical analysis}

The preclinical and clinical datasets were each separated into two subsets: one subset with low fat fraction ( $\mathrm{LFF}, \mathrm{FF}<15 \%$ ) and the other with high fat fraction (HFF, FF $\geq 15 \%$ ). The preclinical (clinical) HFF subset included twenty-five (five) spectra, with a mean FF value of $26.9 \pm 5.5 \%$ $(27.6 \pm 7.8 \%)$. The preclinical (clinical) LFF subset included thirteen (six) spectra, with a mean $\mathrm{FF}$ value of $1.6 \pm 0.5 \%(6.0 \pm 3.5 \%)$.

In the preclinical HFF subset $(n=25)$, an excellent quantification of the major lipid resonance (Lip13) was obtained in all cases with the CRLB values in the range of $1-2 \%$, because of the large fat fraction. The CRLB values of the Lip13 resonance were not improved (that is, the CRLB did not decrease) in spectra acquired with water suppression. With respect to the smaller lipids resonances (Lip21, etc.) a good quantification was also obtained because of the high fat content in the mice of this subset. For instance, the Lip21 resonance is important in lipids quantification to differentiate saturated and unsaturated fatty acids [18] (SFA and UFA, respectively). An example of the individual values of the CRLBs for the Lip21 resonance in each mouse is shown in Table 1. Here, an effect of the water suppression on the CRLB was observed, with an overall increase of the CRLB in spectra acquired with water suppression (increase of $14 \%$ to $80 \%$, in 20 out of 25 mice). In the remaining cases, a small decrease, or no change, in the CRLB values was observed in spectra acquired with water suppression. 


\begin{tabular}{lcccc}
\hline & CRLB(\%) noWS & CRLB(\%) WS & $\Delta$ CRLB(\%) & FF(\%) \\
\hline 1 & 5 & 2 & -150 & 19.9 \\
2 & 6 & 4 & -50 & 24.5 \\
3 & 2 & 2 & 0 & 32.3 \\
4 & 3 & 3 & 0 & 26.4 \\
5 & 6 & 7 & +14 & 17.6 \\
6 & 3 & 4 & +25 & 29.8 \\
7 & 3 & 4 & +25 & 19.7 \\
8 & 3 & 4 & +25 & 21.7 \\
9 & 3 & 4 & +25 & 25.5 \\
10 & 2 & 3 & +33 & 26.5 \\
11 & 4 & 7 & +43 & 26.4 \\
12 & 3 & 6 & +50 & 28.2 \\
13 & 2 & 4 & +50 & 30.3 \\
14 & 2 & 4 & +50 & 30.1 \\
15 & 2 & 4 & +50 & 30.9 \\
16 & 7 & 16 & +56 & 29.8 \\
17 & 4 & 9 & +56 & 30.3 \\
18 & 2 & 5 & +60 & 15.0 \\
19 & 2 & 6 & +67 & 34.8 \\
20 & 1 & 3 & +67 & 25.1 \\
21 & 1 & 3 & +67 & 36.5 \\
22 & 2 & 9 & +78 & 27.2 \\
23 & 2 & 9 & +78 & 36.1 \\
24 & 1 & 5 & +80 & 22.0 \\
25 & 1 & 5 & +80 & 35.5 \\
\hline & & & &
\end{tabular}

Table 1: Cramér-Rao Lower Bound (CRLB) values of the Lip21 resonance (2.02 ppm). MR spectra were acquired with (WS) and without (noWS) water suppression in mice ( $n=25)$ with a hepatic fat fraction $\geq 15 \%$. Overall, an increase of the CRLB values of the Lip21 resonance is observed in the spectra acquired with water suppression. The percentage difference between the CRLB with and without water suppression was calculated as $\triangle C R L B=100 *\left[C R L B_{W S}-C R L B_{n o W S}\right] / C R L B_{W S}$. The last column shows the fat fraction calculated for each spectrum. The increase of CRLB values with water suppression indicates that the best acquisition strategy consists in acquiring MR spectra without water suppression.

In the preclinical LFF subset $(n=13)$, the changes in the CRLB of the Lip13 resonance between spectra acquired with and without water suppression did not exhibit a clear pattern (Table 2). In 50\% of the cases, a degradation of the quantification (i.e., higher CRLBs) was observed in spectra acquired with water suppression. In the other $50 \%$ of cases, the opposite was observed. The Lip21 resonance could not be reliably quantified (CRLB $>10 \%)$ because of the low amount of fat. It should be noted that, in the preclinical LFF subset, the number of averages used for the acquisitions $(\mathrm{NA}=256)$ with water suppression was higher than in the case of the acquisitions $(\mathrm{NA}=64)$ without water suppression; this could explain the lack of differences observed in the CRLBs. 


\begin{tabular}{|c|c|c|c|c|}
\hline & CRLB(\%) noWS & CRLB(\%) WS & $\Delta$ CRLB(\%) & FF(\%) \\
\hline 1 & 20 & 5 & -300 & 0.5 \\
\hline 2 & 7 & 3 & -133 & 2.0 \\
\hline 3 & 4 & 3 & -33 & 1.7 \\
\hline 4 & 5 & 4 & -25 & 1.4 \\
\hline 5 & 4 & 4 & 0 & 1.9 \\
\hline 6 & 5 & 5 & 0 & 0.8 \\
\hline 7 & 3 & 3 & 0 & 1.5 \\
\hline 8 & 2 & 3 & +33 & 2.2 \\
\hline 9 & 6 & 9 & +33 & 1.4 \\
\hline 10 & 3 & 6 & +50 & 1.6 \\
\hline 11 & 2 & 4 & +50 & 1.9 \\
\hline 12 & 3 & 10 & +70 & 1.5 \\
\hline 13 & 4 & 13 & +70 & 2.0 \\
\hline
\end{tabular}

Table 2: Cramér-Rao Lower Bound (CRLB) values of the Lip13 resonance (1.3 ppm). MR spectra were acquired with (WS) and without (noWS) water suppression in mice (n=13) with a small hepatic fat fraction. The percentage difference between the CRLB with and without water suppression was calculated as $\triangle C R L B=$ $100 *\left[C R L B_{W S}-C R L B_{n o W S}\right] / C R L B_{W S}$. The last column shows the fat fraction calculated for each spectrum.

A similar analysis of the effect of water suppression on the lipid quantification was performed on the clinical dataset. For the clinical HFF subset $(n=5)$, an excellent quantification of the major lipid resonance (Lip13) was obtained in spectra acquired with and without water suppression, with the CRLB values in the range of $1-2 \%$ - except for one case $(\mathrm{CRLB}=9 \%)$. In three cases, no changes were observed with water suppression. In two cases, the CRLB values were decreased with water suppression (from $2 \%$ to $1 \%$, and from $9 \%$ to $4 \%$ ). Concerning the lipid resonances other than the Lip13 resonance, it was possible to quantify reliably $(\mathrm{CRLB}<10 \%)$ the Lip21 resonance in both spectra acquired with and without water suppression in four out of five patients. The CRLB value range was $2-6 \%$ and $2-10 \%$ in spectra acquired with and without water suppression, respectively.

In the clinical LFF subset $(\mathrm{n}=6)$, the Lip13 was well quantified, in both spectra acquired with and without water suppression. The range of the CRLBs obtained with and without water suppression was 1-4\% and 2-5\%, respectively. Overall, the water suppression had a negligible effect on the quantitation of the Lip13 resonance: a maximum of $2 \%$ of absolute decrease was found in the CRLB with water suppression in only one case.

In summary, the analysis of CRLB values shows that the strategy of using water suppression for liver MR spectroscopy overall involves either no improvement, or a degradation of the spectral data.

The effect of the water suppression on the Lip53+52 resonance (at $5.3 \mathrm{ppm}$ ) is illustrated by the calculation of the parameter R, defined in the Material and Methods section. For preclinical data, the parameter $\mathrm{R}$ was evaluated for the spectrum with and without water suppression. In all cases, the $\mathrm{R}$ value was higher for the spectrum acquired with water suppression (Figure 3). Similar results were obtained for the clinical data. 


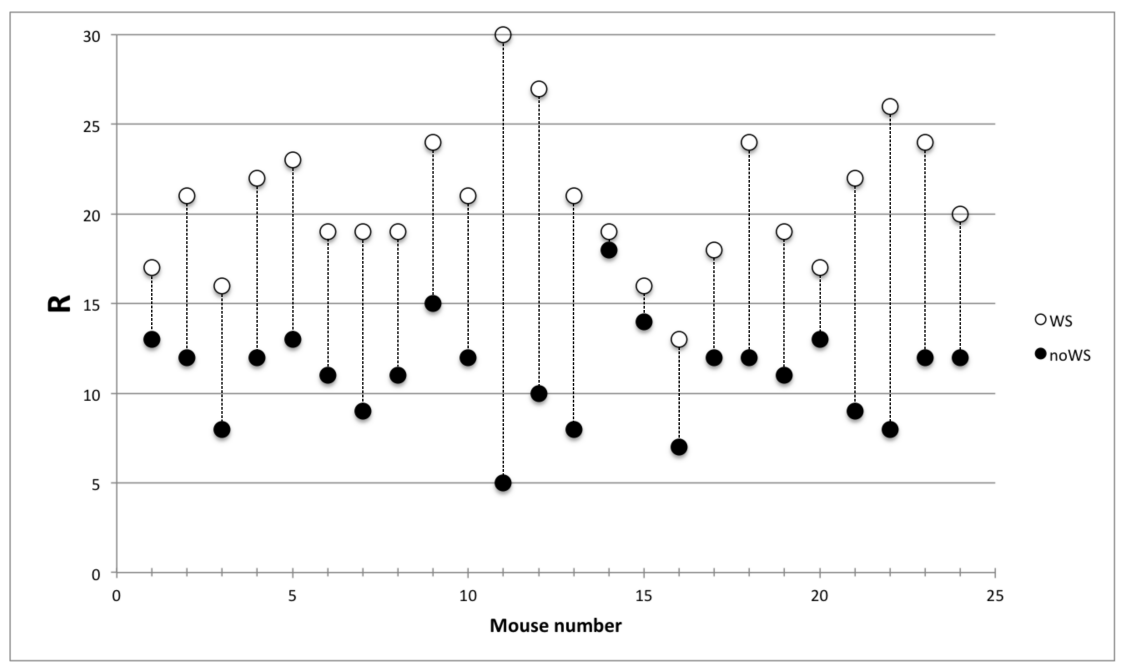

Figure 3: Graphical representation of the ratio $R(R=[$ Lip13]/[Lip53+52]) calculated from spectra acquired on mice presenting a high hepatic fat fraction ( $\geq 15 \%)$ with (WS) or without (noWS) water suppression. This parameter was calculated only for spectra presenting a CRLB<10\% for the Lip53+52 (5.3 ppm) resonance (n=24). The connecting lines have been drawn to aid visual comparison.

For preclinical data (Table 3), in the HFF subset the paired t-test highlighted a strong correlation between the strategy to acquire MR spectra with water suppression and a degradation of the quantification of the lipid resonances (p-value range: 0.0002-0.0392), with the only exception of the Lip53+52 resonance $(\mathrm{p}$-value $=0.0026)$. In the case of the LFF subset, no statistically significant difference was found between the lipid resonances quantification obtained from spectra acquired with or without water suppression (p-values of 0.30 and 0.85 for Lip09 and Lip13 resonances, respectively).

\begin{tabular}{|c|c|c|c|c|}
\hline & \multicolumn{2}{|c|}{$\begin{array}{c}\mathrm{HFF} \\
\mathrm{n}=25\end{array}$} & \multicolumn{2}{|c|}{$\begin{array}{c}\mathrm{LFF} \\
\mathrm{n}=13\end{array}$} \\
\hline & WS & noWS & WS & noWS \\
\hline Lip09 & & $0.0022^{* *}$ & & 0.3094 \\
\hline Lip13 & & $0.0042^{* *}$ & & 0.8499 \\
\hline Lip16 & & $0.0392^{*}$ & & \\
\hline Lip21 & & $0.0002^{* *}$ & & \\
\hline Lip23 & & $0.0116^{*}$ & & \\
\hline Lip $53+52$ & $0.0026^{* *}$ & & & \\
\hline
\end{tabular}

Table 3: P-values of paired t-test between the CRLB of each lipid resonance calculated from spectra acquired with (WS) or without (noWS) water suppression. MR spectra were acquired in mice with a high fat fraction $(\geq 15 \%)$ and in mice a low fat fraction $(<15 \%)$. The CRLB of lipid resonances are always lower without water suppression, except for the Lip53+52 resonance. $\left({ }^{* *}\right)=$ p-value $<0.01 ;\left(^{*}\right)=p$-value $<0.05$.

For clinical data, in the HFF subset there was no statistically significant difference between the Lip13 resonance quantification obtained from spectra acquired with or without water suppression (pvalues of 0.14). The same result applied to the quantification of the Lip21 resonance (p-value of 0.27). In the LFF subset, for the Lip13 (the only lipid resonance that could be reliably quantified) the paired 
t-test showed a correlation between the strategy to acquire MR spectra with water suppression and an improvement in the quantification of the lipid resonances ( $\mathrm{p}$-value $=0.006$ ). However, since the CRLB values are similar in both strategies, we can consider that this improvement is negligible. In summary, the statistical analysis of our results shows that the strategy of using water suppression for liver MR spectroscopy involves either a deterioration of spectral data or, in best case scenario, no improvement.

\section{Discussion}

In the current study, a quantitative assessment of the impact of water suppression on the hepatic lipid quantification was performed. To date, in both preclinical and clinical studies there is no established approach with respect to the data acquisition of MR spectra in liver. In particular, to determine the fat percentage and composition, the experimental protocol includes in some cases two MR spectra (one with and the other without water suppression) $[3,9,14,18,20,22-25]$ and in other cases only one MR spectrum (without water suppression) [?,2,4,6,8,19,26-32] . In the current study, MR spectra with and without water suppression were acquired in patients, at $3 \mathrm{~T}$, and in mice, at the moderate field of $4.7 \mathrm{~T}$. The CRLB of each lipid resonance, evaluated by the LCModel, was used as a metric to determine the effect of water suppression on the hepatic lipid quantification. The CRLBs obtained from the analysis of spectra acquired with water suppression were compared to those obtained from spectra without water suppression. The analysis of CRLB of the lipid resonances showed that there is no benefit in acquiring the MR spectrum with water suppression since it does not improve the quantification of these resonances. In addition, water suppression could result in degradation of the lipid resonance nearby the water resonance. Thus, the results of the current study indicate that -for the experimental conditions of the current work - it is possible to shorten the experimental protocol without loss of information by not acquiring an additive MR spectrum with water suppression.

It should be noted that the experimental conditions of this study - in particular the magnetic field strength- are similar to those of previous studies performed in clinical settings, where the $3 \mathrm{~T}$ MRI systems are becoming increasingly common [6], and in preclinical models. Interestingly, many preclinical works in mouse and rat liver have been performed at moderate magnetic field strengths $[18,26,29,33]$. This is in stark contrast to MR spectroscopy of brain where the majority of the preclinical studies is performed at magnetic field strength $\geq 7 \mathrm{~T}$ [14]. Thus, the results of the current study are relevant and of interest to the MR scientists working on liver spectroscopy both in clinical and preclinical models.

With respect to the data analysis of in vivo MR spectra of hepatic lipids, different dedicated software and models are currently used, without a well-established method. This is due to the fact that the precise line shape of the lipid resonances is not known a priori. As a matter of fact, given the complex structure of the triglyceride molecule, quantum mechanics simulations based on the density matrix formalism can model the spectral shape of lipid resonances only to a certain extent. As a consequence, current fitting methods employ empirical approaches. In particular, the software used in the current study (LCModel) employs a combination of Gaussians and Lorentzians for each lipid resonance to determine the area under the resonance peak. It should be noted here that a different scenario char- 
acterizes the data analysis of MR spectra acquired in organs other than liver. In MRS of brain and prostate, for instance, it is possible to model the line shapes corresponding to each metabolite (glutamate, myoinositol citrate, etc.) with a high level of precision using quantum mechanics simulations; as a result, it is possible to use this information as a priori knowledge in data fitting.

One limitation of the current study is that, to assess the impact of water suppression on hepatic lipid quantification, the only metric employed was the CRLB values. The limitations of the CRLB values, well known and nicely described in the literature [34,35], might have an impact on the assessment of the lipid quantification in the low fat fraction scenario. On the other hand, there is no established metric available to validate the CRLB results for the two specific resonances (Lip13 and Lip09) that have been quantified in the low fat fraction cases; further studies would be needed to elucidate this issue, with a specific, tailored metric.

The water suppression module that is typically used on clinical scanners consists of 2 to 4 CHESS (CHemical Shift-Selective) pulses, whereas more sophisticated approaches are available on preclinical scanners. The water suppression module employed in the current study on the clinical scanner was WET with 3 CHESS pulses, and on the preclinical scanner was VAPOR with 7 CHESS pulses. For the MR data acquisition performed on the clinical scanner, no manual adjustment of the water suppression was done. As a result, incomplete water suppression was observed in some spectra. On the contrary, in the preclinical experiment the use of VAPOR -in addition to the manual adjustment of the water suppression pulse gains - resulted in excellent water suppression. It should be noted that in most clinical studies, due to time constraints, manual adjustments -if available- are rarely performed. In the current study, a systematic effect was observed on the quantification of the Lip53+52 resonance in the MR spectra acquired with and without water suppression, on mice. The parameter R, defined in the Material and Methods section, was used to quantify this effect. The value of $\mathrm{R}$ with water suppression was lower than the value of $\mathrm{R}$ without water suppression. Since there is no ground truth for the value of the Lip53+52 resonance in our experimental conditions, from the current data it is not possible to determine which is the best approach to quantify this resonance. As mentioned previously, when considering the literature regarding preclinical studies performed at similar field strength (3 T4.7 T), there is no established method of data acquisition (with or without water suppression) for the lipid quantification. In some preclinical studies, MR spectra were acquired with water suppression, however the Lip53+52 resonance was not used in the quantification, since it could have been partially suppressed by the water suppression [33]; in other studies, on the other hand, no water suppression was used [23-27]. Based on the results of the current study, it is not clear which is the most beneficial approach for the quantification of the Lip53+52, at the moderate field of $4.7 \mathrm{~T}$.

In clinical studies of liver pathologies the biomarker of interest is the fat content, which can be determined using the major lipid resonance at $1.3 \mathrm{ppm}$. For instance, a biomarker for the diagnosis of steatosis is based on a $5 \%$ fat content threshold. The hepatic lipid composition is rarely assessed; however, the quantification of hepatic lipid composition could be of great interest to gain insight into the lipid metabolism in liver. Intuitively, it might seem natural to acquire MR spectra in liver with the water suppression, as it is done in MRS of brain, to improve the detection of the smaller lipid resonances. The results of the current study show that there is no improvement in the quantification of lipid resonances by employing the water suppression; this applies to the quantification of both 
fat content as well as fat composition. As a consequence, the clinical protocol can be shortened by acquiring only the non-water-suppressed MR spectrum.

\section{Conclusion}

The results of the current study indicate that, for quantification of lipid resonances in liver, it is advantageous to perform MR spectroscopy without water suppression. This applies to the quantification of fat fraction as well as fat composition, both in preclinical and clinical scenario (at moderate fields). As a consequence, the preclinical and clinical protocols can be shortened by only acquiring an MR spectrum without water suppression.

\section{Appendix}

An additional data analysis of the MR spectra was performed in order to provide further insight into the current study. In the preclinical (clinical) HFF subset, the mean value of the full width at half maximum (FWHM) of the water resonance was $53 \pm 7 \mathrm{~Hz}(46 \pm 18 \mathrm{~Hz})$. In the preclinical (clinical) LFF subset, the mean value of the FWHM of the water resonance was $59 \pm 13 \mathrm{~Hz}(32 \pm 9 \mathrm{~Hz})$. The FWHM observed in the preclinical HFF and LFF subsets is in agreement with the FWHM value found in a previous study performed at $4.7 \mathrm{~T}$ on mice [18]. The FWHM observed on the clinical HFF subset is higher than the value ( $\mathrm{FWHM} \approx 30 \mathrm{~Hz}$ ) found in a previous study at $3 \mathrm{~T}$ on patients [32]. On the other hand, the FWHM observed on the clinical LFF subset is in agreement with the value found in Ref. [32].

The noise level measured on the preclinical HFF subset was $163 \pm 31$ (a.u.) without water suppression and $179 \pm 44$ (a.u.) with water suppression. Thus, it appears that the VAPOR water suppression did not affect the noise level.

The quantification of the lipid composition was calculated using the Corbin et al. algorithm [18] on the HFF subsets (preclinical and clinical) with and without water suppression. It should be pointed out that in the Corbin method, the UFA fraction is calculated as: $100 * 3 / 4 *[$ Lip21]/[Lip09] and the SFA fraction is calculated as: $100-U F A$. On the preclinical HFF subset, the results were of SFA=29.23\% and $\mathrm{UFA}=70.77 \%$ (in both cases the standard deviation was of $4.6 \%$ ) without water suppression. With water suppression the results were $\mathrm{SFA}=30.88 \%$ and $\mathrm{UFA}=69.12 \%$ (in both cases the standard deviation was of 5.29\%). On the clinical HFF subset, the quantification of the lipid composition yielded the values of $\mathrm{SFA}=43.25 \%$ and $\mathrm{UFA}=56.75 \%$ (standard deviation $=6.08 \%$ ) without water suppression and $\mathrm{SFA}=39.65 \%$ and $\mathrm{UFA}=60.35 \%$ (standard deviation $=5.60 \%$ ) with water suppression. Thus, the values of saturated and unsaturated fatty-acid fractions obtained from spectra with water suppression were comparable to those from spectra without water suppression, both in clinical and preclinical data. 


\section{Acknowledgements}

We are most grateful to the PRISM core facility (Biogenouest@C, UMS Biosit, Université de Rennes 1

- Campus de Villejean, 35043 Rennes Cedex, France) for its technical support. 


\section{References}

[1] Fabbrini, E., Sullivan, S., and Klein, S. Obesity and nonalcoholic fatty liver disease: biochemical, metabolic, and clinical implications. Hepatology (2010). 51(2):679-689.

[2] Cowin, G.J., Jonsson, J.R., Bauer, J.D., Ash, S., Ali, A., Osland, E.J., Purdie, D.M., Clouston, A.D., Powell, E.E., and Galloway, G.J. Magnetic resonance imaging and spectroscopy for monitoring liver steatosis. Journal of Magnetic Resonance Imaging (2008). 28(4):937-945.

[3] Johnson, N.A., Walton, D.W., Sachinwalla, T., Thompson, C.H., Smith, K., Ruell, P.A., Stannard, S.R., and George, J. Noninvasive assessment of hepatic lipid composition: advancing understanding and management of fatty liver disorders. Hepatology (2008). 47(5):1513-1523.

[4] Szczepaniak, L.S., Nurenberg, P., Leonard, D., Browning, J.D., Reingold, J.S., Grundy, S., Hobbs, H.H., and Dobbins, R.L. Magnetic resonance spectroscopy to measure hepatic triglyceride content: prevalence of hepatic steatosis in the general population. American Journal of PhysiologyEndocrinology and Metabolism (2005). 288(2):E462-E468.

[5] Machann, J., Stefan, N., and Schick, F. 1 H MR spectroscopy of skeletal muscle, liver and bone marrow. European journal of radiology (2008). 67(2):275-284.

[6] Hamilton, G., Yokoo, T., Bydder, M., Cruite, I., Schroeder, M.E., Sirlin, C.B., and Middleton, M.S. In vivo characterization of the liver fat $1 \mathrm{H}$ MR spectrum. NMR in Biomedicine (2011). 24(7):784-790.

[7] Thomsen, C., Becker, U., Winkler, K., Christoffersen, P., Jensen, M., and Henriksen, O. Quantification of liver fat using magnetic resonance spectroscopy. Magnetic resonance imaging (1994). 12(3):487-495.

[8] Thomas, E.L., Hamilton, G., Patel, N., O’Dwyer, R., Doré, C.J., Goldin, R.D., Bell, J.D., and Taylor-Robinson, S.D. Hepatic triglyceride content and its relation to body adiposity: a magnetic resonance imaging and proton magnetic resonance spectroscopy study. Gut (2005). 54(1):122127.

[9] Ye, Q., Danzer, C.F., Fuchs, A., Vats, D., Wolfrum, C., and Rudin, M. Longitudinal evaluation of hepatic lipid deposition and composition in ob/ob and ob/+ control mice. NMR in Biomedicine (2013). 26(9):1079-1088.

[10] Reeder, S.B., Cruite, I., Hamilton, G., and Sirlin, C.B. Quantitative assessment of liver fat with magnetic resonance imaging and spectroscopy. Journal of magnetic resonance imaging (2011). 34(4):729-749.

[11] Provencher, S.W. Estimation of metabolite concentrations from localized in vivo proton NMR spectra. Magnetic Resonance in Medicine (1993). 30(6):672-679.

[12] Hennig, J., Nauerth, A., and Friedburg, H. RARE imaging: a fast imaging method for clinical MR. Magnetic Resonance in Medicine (1986). 3(6):823-833. 
[13] Bottomley, P.A. Selective volume method for performing localized NMR spectroscopy (1984). US Patent 4,480,228.

[14] Tkáč, I., Starčuk, Z., Choi, I.Y., and Gruetter, R. In vivo 1H NMR spectroscopy of rat brain at 1 ms echo time. Magnetic Resonance in Medicine (1999). 41(EPFL-ARTICLE-177519):649-656.

[15] Kiefer, B., Grassner, J., and Hausman, R. Image acquisition in a second with half Fourier acquisition single-shot turbo spin-echo. J Magn Reson Imaging (1994). 4(Suppl):86-87.

[16] Frahm, J., Merboldt, K.D., and Hänicke, W. Localized proton spectroscopy using stimulated echoes. Journal of Magnetic Resonance (1969) (1987). 72(3):502-508.

[17] Ogg, R.J., Kingsley, R., and Taylor, J.S. WET, a T 1-and B 1-insensitive water-suppression method for in vivo localized 1 H NMR spectroscopy. Journal of Magnetic Resonance, Series B (1994). 104(1):1-10.

[18] Corbin, I.R., Furth, E.E., Pickup, S., Siegelman, E.S., and Delikatny, E.J. In vivo assessment of hepatic triglycerides in murine non-alcoholic fatty liver disease using magnetic resonance spectroscopy. Biochimica et Biophysica Acta (BBA)-Molecular and Cell Biology of Lipids (2009). 1791(8):757-763.

[19] Strobel, K., van den Hoff, J., and Pietzsch, J. Localized proton magnetic resonance spectroscopy of lipids in adipose tissue at high spatial resolution in mice in vivo. Journal of lipid research (2008). 49(2):473-480.

[20] Cheung, J.S., Fan, S.J., Gao, D.S., Chow, A.M., Yang, J., Man, K., and Wu, E.X. In vivo lipid profiling using proton magnetic resonance spectroscopy in an experimental liver fibrosis model. Academic radiology (2011). 18(3):377-383.

[21] Cavassila, S., Deval, S., Huegen, C., Van Ormondt, D., and Graveron-Demilly, D. Cramer-Rao bounds: an evaluation tool for quantitation. NMR in Biomedicine (2001). 14(4):278-283.

[22] van Werven, J.R., Marsman, H.A., Nederveen, A.J., Smits, N.J., ten Kate, F.J., van Gulik, T.M., and Stoker, J. Assessment of Hepatic Steatosis in Patients Undergoing Liver Resection: Comparison of US, CT, T1-weighted Dual-Echo MR Imaging, and Point-resolved 1H MR Spectroscopy 1. Radiology (2010). 256(1):159-168.

[23] Rijzewijk, L.J., van der Meer, R.W., Lubberink, M., Lamb, H.J., Romijn, J.A., de Roos, A., Twisk, J.W., Heine, R.J., Lammertsma, A.A., Smit, J.W., et al. Liver fat content in type 2 diabetes: relationship with hepatic perfusion and substrate metabolism. Diabetes (2010). $\mathbf{5 9}(11): 2747-2754$.

[24] Kim, H., Taksali, S.E., Dufour, S., Befroy, D., Goodman, T.R., Petersen, K.F., Shulman, G.I., Caprio, S., and Constable, R.T. Comparative MR study of hepatic fat quantification using single-voxel proton spectroscopy, two-point dixon and three-point IDEAL. Magnetic Resonance in Medicine (2008). 59(3):521-527. 
[25] Petersen, K.F., Dufour, S., Savage, D.B., Bilz, S., Solomon, G., Yonemitsu, S., Cline, G.W., Befroy, D., Zemany, L., Kahn, B.B., et al. The role of skeletal muscle insulin resistance in the pathogenesis of the metabolic syndrome. Proceedings of the National Academy of Sciences (2007). 104(31):12587-12594.

[26] Marsman, H.A., van Werven, J.R., Nederveen, A.J., Ten Kate, F., Heger, M., Stoker, J., and van Gulik, T.M. Noninvasive quantification of hepatic steatosis inrats using $3.0 \mathrm{~T}$ 1H-magnetic resonance spectroscopy. Journal of Magnetic Resonance Imaging (2010). 32(1):148-154.

[27] Peng, X.G., Ju, S., Qin, Y., Fang, F., Cui, X., Liu, G., Ni, Y., and Teng, G.J. Quantification of liver fat in mice: comparing dual-echo Dixon imaging, chemical shift imaging, and 1H-MR spectroscopy. Journal of lipid research (2011). 52(10):1847-1855.

[28] Hájek, M., Dezortová, M., Wagnerová, D., Škoch, A., Voska, L., Hejlová, I., and Trunečka, P. MR spectroscopy as a tool for in vivo determination of steatosis in liver transplant recipients. Magnetic Resonance Materials in Physics, Biology and Medicine (2011). 24(5):297-304.

[29] Heger, M., Marsman, H.A., Bezemer, R., Cloos, M.A., van Golen, R.F., and van Gulik, T.M. Non-invasive quantification of triglyceride content in steatotic rat livers by 1 H-MRS: when water meets (too much) fat. Academic radiology (2011). 18(12):1582-1592.

[30] Pineda, N., Sharma, P., Xu, Q., Hu, X., Vos, M., and Martin, D.R. Measurement of Hepatic Lipid: High-Speed T2-Corrected Multiecho Acquisition at 1H MR Spectroscopy-A Rapid and Accurate Technique 1. Radiology (2009). 252(2):568-576.

[31] Gambarota, G., Tanner, M., van der Graaf, M., Mulkern, R.V., and Newbould, R.D. 1H- MRS of hepatic fat using short TR at 3T: SNR optimization and fast T2 relaxometry. Magnetic Resonance Materials in Physics, Biology and Medicine (2011). 24(6):339-345.

[32] Krššák, M., Hofer, H., Wrba, F., Meyerspeer, M., Brehm, A., Lohninger, A., Steindl-Munda, P., Moser, E., Ferenci, P., and Roden, M. Non-invasive assessment of hepatic fat accumulation in chronic hepatitis $\mathrm{C}$ by $1 \mathrm{H}$ magnetic resonance spectroscopy. European journal of radiology (2010). 74(3):e60-e66.

[33] van Werven, J.R., Marsman, H.A., Nederveen, A.J., Fiebo, J., van Gulik, T.M., and Stoker, J. Hepatic lipid composition analysis using 3.0-T MR spectroscopy in a steatotic rat model. Magnetic resonance imaging (2012). 30(1):112-121.

[34] Kreis, R. and Boesch, C. Bad spectra can be better than good spectra. In ISMRM 11th scientific meeting, Toronto, Ontario, Canada (2003) 264.

[35] Kreis, R. Issues of spectral quality in clinical 1H-magnetic resonance spectroscopy and a gallery of artifacts. NMR in Biomedicine (2004). 17(6):361-381. 